\title{
An Interaction-Oriented Model of Customer Behavior for the Simulation of Supermarkets
}

\author{
Yoann Kubera, Philippe Mathieu, Sébastien Picault \\ LIFL, University Lille 1 - 59655 Villeneuve d'Ascq, France \\ Email: firstname.surname@lift.fr
}

\begin{abstract}
Since several years, great distribution firms implement more and more complex layout and shelf allocation strategies, so as to force empirical know-how to combine with Artificial Intelligence tools. Thus simulation has become an essential tool for designing efficient article layouts. Mathematical models based on statistical observations have been replaced by agent-based models. In this paper we argue that the modelling of individual behaviors of customers in a supermarket must be done through interaction-oriented approaches, in order to allow large-scale simulations and flexible models. Our model, based on our IODA methodology, is part of a "Serious Game" dedicated to vendor training. It takes into account the diversity of customer preferencies, the location of articles, and the side-effects of customer moves, so as to measure the consequence of management choices on the global outcomes of the supermarket.
\end{abstract}

Keywords-Supermarket Simulation, Individual-Based Models Design, Interactions, Serious Game

\section{INTRODUCTION}

From small groceries to hypermarket, the allocation of products to shelves, as well as the general layout of the store, has a direct impact on the behavior of consumers. Since several years, automation has provided a set of tools that facilitate the design and the management of supermarkets. From handmade, empirical layouts to planograms, then to stochastic simulation, supermarket engineering has now come to agent-based models in order to anticipate the consequences of minor organizational changes on the consuming behaviors.

Our aim in this paper is to give a survey of the classical modelling approaches, and show that there is an increasing need for individual-based models. The very first stage consists of mathematical or statistical models; then, we present agent-based approaches which do not take spatial parameters into account. Finally, we present our own contribution to this modelling field, through an application based on a generic, flexible interaction-oriented methodoloy.

\section{From StatisticAl to AGENT-BASED MODELS}

A first study of the factors that influence the behavior of customers, relies upon data collected in actual stores, e.g. through classical data mining techniques, at a macroscopic level. Such statistics are used to identify "customer profiles" (statistical clusters), or to extrapolate from the current management to alternative situations, or to validate the simulations (by comparing predicted results to other actual situations).

For instance, regression analysis techniques [1] lead to reliable quantitative results, but are confined to linear dependencies ; in addition they cannot take many factors into account. Other approaches include multi-layer neural networks [1], which allow then to deal with non-linear dependencies; however, they are not well adapted to treat large numbers of factors, because of the rise in complexity and the possible occurrence of local minima.

Numerical simulations have also been proposed, e.g. through Monte-Carlo techniques [2], or in discrete event simulations (e.g. Promodel [3]). The general principle consists of using the statistics as a probabilistic model for future behavior, based on mathematical expectations.

However, mere numerical approaches can only play a predictive role. Thus, if the parameter space is very large, it has to be explored completely to find the best set of parameters. That would not be the case by using an explanatory model, i.e. a model that gives information about the processes that lead to specific results. In order to gain better understanding of the interactions that lead to global data, agent-based models have thus been introduced in simulations.

A first step to take more parameters into account consists of using agents for introducing a realistic model of individual customers based on psychological studies (e.g. [5] or [4]). However, it raises very difficult issues regarding the validation of the proposed models, and does not fit the 'Occam's razor' principle, since the correct behavior might be obtained through more simple individual agents.

A second agent approach consists in "agentifying" customer profiles with agents or group of agents, e.g. through holonic models [6]. Customer profiles, built through the analysis of gathered real data, are used to determine the parameters in the individual agents. Then the agents are able to show the amount of products they purchase. This allows the exploration of a large number of parameters, and of course the handling of non-linear dependencies. Individual agents make their choices according to Bayesian nets (drawn from actual data), and thus are able to provide correct quantitative predictions.

Though, such holonic systems are not fully relevant to 
model supermarkets. Indeed, the multiple consumer profiles can be classified into groups, but those groups are just empirical clusters that should not be used as "ideal" categories in the model. In fact, such categories must be understood as mere emergent outcomes that are the product of individual preferences and a spatial organization.

As a matter of fact, planograms (i.e. dedicated diagrams) have been widely used in early analogical simulations to anticipate, in an empirical way, the consequences of product placement on the behavior of consumers [7]. They are usually produced by many professional softwares, among which some allow a 3D-rendering of the shelves and virtual visits. As such, those tools do not allow any simulation involving realistic agents. However, the intensive use of planograms is a good clue about the necessity to take space into account in simulations. This leads us to consider simulations involving situated agents, i.e. where the organization of space, and the fact that the behaviors of the agents rely on spatial features of the environment, play a crucial role on emerging results.

SimStore [8] for instance is very close to our own purpose: the clients are represented by autonomous agents, provided with shopping lists, and walking around in the store to find their products. Casti emphasizes the crucial issue of paths which are subject to a dynamic tradeoff between the aims of the store manager (to encourage long paths for complete exploration) and those of the clients (to find the most efficient way to carry out all purchase in the shortest time).

We completely agree with the aims and principles of such a method. It is a bottom-up approach, since the global results (purchases, paths, etc.) are produced by the spatial activity of all agents. We think that it is the only reliable way to model artificial customers in an artificial store in flexible situations. However, SimStore cannot easily extend to large-scale simulation, since the design of behaviors is tightly coupled with the architecture of agents. For the same reasons, the behaviors are designed for the very specific context of store simulations, and thus have no genericity nor reusability.

Thus, a better way to model individual-based behaviors in a reusable, sustainable and scalable way, requires a separate implementation of, on the one hand, a general simulation engine, and on the other hand, the domain-related knowledge. In addition, the latter must be split itself in two software libraries: $1^{\circ}$ the features of the agents which represent the entities that take part to the simulation, and $2^{\circ}$ the definition of available behaviors, as interactions that can occur between agents: thus, in order to represent the relationships that exist between the relevant entities. This is what we call an interaction-oriented approach [9], and will be described in the next section.

\section{AN INTERACTION-ORIENTED MODEL}

\section{A. IODA: The Interaction-Oriented Approach}

The main idea in our Interaction-Oriented approach, called IODA $^{1}$, consists of providing the interactions that occur in an individual-based model with a concrete and independent software implementation, so that separate agent libraries and interaction libraries can be built gradually from a specific application domain. Formal definitions of IODA concepts are provided in [9].

In the IODA approach, all relevant entities are represented by "agents" [10], and all behaviors that some of those entities exhibit are represented by "interactions". This provides indeed an homogeneous description of entities and behaviors.

An Interaction is a structured set of action primitives involving two agents, which can occur when a trigger (implicit or explicit goal) and a condition (logical or physical prerequisite for actions), based upon perception primitives, are met. Those perception and action primitives are functions or procedures that are implemented in the actual agents, with respect to their specificities (e.g. more or less cognitive).

Agents involved in an interaction generally do not play the same role. The agent that can perform the interaction is called Source, while the agent that can undergo the interaction is called Targets.

A simulation model is mainly defined through the way interactions are assigned to source or target agent families: this assignation is done through the interaction matrix. At runtime, an interaction occurs when target agents are present into the neighborhood of the source agent, within a limit distance, in order to assess that the source agent is "close enough" to the target. Additionally, every assigned interaction is endowed with a priority, so to build a hierarchy between them from the viewpoint of the source agent.

A simple interaction matrix we used in the context of supermarket simulation is shown on figure 1 (other examples are provided on: http://www. lifl.fr/SMAC/projects/ioda/)

All IODA agents compute their behavior according to the same generic process, which aims at selecting an interaction/target pair among the interactions that the agent can perform and the neighbors that can be used as target. Thus each potential source agent has to test the conditions of the interactions it can perform with its neighbors, with respect to the priority level.

\section{B. The agents of the IODA model}

The model we present below is part of a "Serious Game" simulator, the "FormatStore Project", which has been designed together with Idees-3Com, a company specialized in $3 \mathrm{D}$ interactive applications, and with Enaco, a business

${ }^{1}$ IODA means "Interaction-Oriented Design of Agent simulations" 
school, so as to provide a tool for students training as salesperson, trade assistant or shop manager.

In the IODA model of a supermarket, all relevant "entities" are represented by agents:

- Consumers: the class Client defines all perception/action primitives that consitute the elementary capacities of clients. Such agents may be very simple or much more sophisticated, as we explain below. They have at least a shopping list with mandatory articles, and may have additional interest articles.

- Articles: We represent a collection of identical articles by a single agent, endowed with the quantity of physical items it represents. Articles have also a price, a quality level, a location and a spatial extent. The article is identified through a 3-level stamp: its category (e.g. drinks), its subcategory (e.g. mineral water) and its brand.

- Entrance areas: these are just agent sources that can be tuned to generate clients in accordance with real, empirical data. Realistic results for arrivals in the supermarket are given by a Poisson distribution. In addition, the clients are generated with typical shopping lists based on real data.

- Checkouts: when clients have collected all their articles, or decide to stop their purchases (e.g. because of time constraints), they enter a queue which counts their articles and destroys them.

- Indication panels: in order to help clients (or influence them) in their purchases, some signs are located at strategical points (entrance, ends of shelves...). In the IODA approach this is done through agents that interact with the clients, providing them with informations (e.g. the category of the articles nearby).

- Supermarket staff (optional): some agents can be added to represent employees if the store, in charge of helping clients, renewing or moving articles, changing prices...

- Shelves (optional): such agents, intended to work as containers for articles, play a role in the structure of the environment. They are used in a few experiments to enhance the study of spatial influences on the purchases.

\section{The interactions}

Figure 1 gives an overview of the interaction matrix that has been used in one of our experiments. It is very easy to modify the features of this matrix, to allow an incremental, flexible ("plug-and-play") design of experiments by combining easily agents and interactions to produce appropriate behavior. The only constraint on agents is that they should implement the primitives required in the interactions that they have to perform or undergo. No particular assumption is made regarding the implementation of those primitives, so that agents can be as complex as needed, as we are going to explain in the next topic.

\section{The integration of psychological knowledge}

It is crucial to understand that, in the IODA approach, agents may use any given interactions library, whatever their cognition level may be. Depending on how primitives are implemented in the agents, cognitive behaviors can be obtained as well as reactive ones. Moreover, heterogeneous agents, i.e. cognitive agents mixed with reactive agents, can be simulated at the same time and can interact through the same interactions.

For exemple, let us consider the following interaction (we suppose that it can be performed by a Client as source on an Article as target):

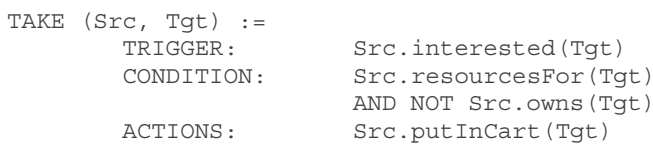

This interaction, as it is written above, represents no more than the fact that, in order to buy article Tgt, client SrC must: 1) be "interested" by this article; 2) have enough ressources (among them, money of course) to buy it; 3 ) not already own an similar article. Thus this is a very abstract definition of the interaction that consists of "taking an article". The designer has still do determine the cognitive level he wants to endow the agents (namely the clients) with. Here are a few examples:

- A pure reactive agent just owns a shopping cart, a shopping list and a budget. Thus, the interested primitive tests if $\mathrm{T} \in$ shopping-list

- A less reactive agent works on two lists: one for mandatory articles, and the other for "interesting" articles with numerical preferencies. In that case, interested returns a decimal value in $[0,1]$ that represents either the fact that Tgt $\in$ mandatory-list, or a weight that reflects the preference for this article and its price.

- A true cognitive agent uses inferences, beliefs, knowledge, planning, etc. In that case the primitives launch inference engines or reflect a mental state: then, interested is written like follows: KNOW(Tgt $\in$ shopping-list) OR BEL(USEFUL(Tgt)) OR (KNOW (LOVE(Tgt)) AND NOT BEL(FAT(Tgt))).

This polymorphism in the actual performance of interactions allow indeed to tune the agent model according to psychological knowledge.

\section{E. Experiments}

At this time, this model has been tested according to two main topics: first, the identification of the simplest set of agents and interactions required for modelling the behavior of consumers in the supermarket; then, the scalability of the approach, regarding large numbers of agents (up to $10^{4}-$ $-10^{5}$ ) and many diverse agent families.

\section{Conclusion}

The research works we have presented here deal with the issue of modelling the behavior of customers in a 


\begin{tabular}{|c|c|c|c|c|c|c|}
\hline source $\quad$ target & $\emptyset$ & Client & Article & Checkout & Entrance & Sign \\
\hline Client & $\begin{array}{c}+(\text { SeekCheckout;5) } \\
+(\text { ChooseNextItem;2) } \\
+(\text { SeekTarget; } 1) \\
+(\text { MoveToTarget;0) }\end{array}$ & $+($ Talk;2;1;1) & $\begin{array}{c}+(\text { Take } ; 4 ; 1 ; 1) \\
+(\text { MoveTowards;3;1;3) }\end{array}$ & $+($ MoveTowards;6;1;5) & & \\
\hline \multicolumn{7}{|l|}{ Article } \\
\hline Checkout & $\begin{array}{l}+(\text { Open; } 2) \\
+(\text { Close } ; 2)\end{array}$ & $+($ DealWith; $1 ; 1 ; 0)$ & & & & \\
\hline Entrance & $+($ CreateClient; 0$)$ & & & & & \\
\hline Sign & & $+($ Inform;1;1;10) & & & & \\
\hline
\end{tabular}

Figure 1. Example of an interaction matrix for a supermarket simulation. The ' $\emptyset$ ' column contains degenerate interactions (target $=$ environment). The integer following the name of the interaction is the priority level (from the viewpoint of the source agent). For non-degenerate interactions, the other numbers represent respectively the cardinality (i.e. number of targets required) and the limit distance (below which the interaction can occur); e.g.: a client can perform Take on an article, with priority 4 , if this article is within 1 distance unit - and of course if the trigger and condition of Take are fulfilled for both client and article.

supermarket. We have shown an increasing need for detailed, individual-based models. Indeed, classical models based on statistical inferences or numerical simulations cannot take into account many parameters in order to anticipate the consequences of management or placement choices upon the purchases of the clients. Agents have been first introduced to represent typical behaviors, observed as clusters in real data. However, situated agents enable much powerful and detailed simulations, but much of the time the design of a simulation is an ad hoc process which lacks reusability and scalability.

Thus, we promote an Interaction-Oriented approach (the IODA methodology) which allows a flexible design of supermarket models. Since our approach makes a clear separation between domain knowledge (structure of the agents, interactions they can perform or undergo) and a generic, multi-purpose simulation engine, it is quite simple to change one parameter independently from the others, to test an hypothesis and reconfigure the layout of the supermarket as in a virtual benchmark, replace the interactions that agents can perform, or build models in an incremental way. In addition, our implementation (the JEDI platform) supports large-scale simulations with about 80000 articles in the supermarket at the same time. Since the supermarket model takes place in participative simulations, some indicators (global revenue, preferred articles...) are available to business students and to their teachers so as to assess their own skill acquisition, enhance their vending methods, or just test some ideas about new strategies.

We currently work, on the one hand, on introducing and tuning some psychological knowledge, and on the other hand, on extending the model for handling large commercial places where several supermarkets compete or complement each other.

\section{ACKNOWLEDGMENT}

The FormatStore Project is supported by a funding from the French Ministry of Research.

\section{REFERENCES}

[1] H.-L. Poh, J. Yao, and T. Jasic, "Neural Networks for the Analysis and Forecasting of Advertising and Promotion Impact". Intelligent Systems in Accounting, Finance and Management, Vol. 7, No. 4, 1998.

[2] C. Baydar, "One-to-One Modeling and Simulation: A New Approach in Customer Relationship Management for Grocery Retail", SPIE Conference on Data Mining and Knowledge Discovery: Theory, Tools, and Technology IV, 2002.

[3] J. A. Alvarado, L. M. Pulido, "Simulation and Experimental Design Applied to Sizing Supermarket Cashiers in Colombia", Winter Simulation Conference, 2008.

[4] M.A. Janssen and W. Jager, "An Integrated Approach to Simulating Behavioural Processes: A Case Study of the Lockin of Consumption Patterns", Journal of Artificial Societies and Social Simulation, 2, No. 2, 1999.

[5] T. Zhang and D. Zhang, "Agent-based simulation of consumer purchase decision-making and the decoy effect", Journal of Business Research, vol. 60, issue 8, Complexities in Markets Special Issue, pp. 912-922, August 2007.

[6] A. Schwaiger and B. Stahmer, "SimMarket: Multiagent-Based Customer Simulation and Decision Support for Category Management", MATES 2003: multiagent system technologies, Springer, LNAI 2831, pp. 74-84, 2003.

[7] R. Bai, E.K. Burke and G. Kendall, "Heuristic, Meta-heuristic and Hyper-heuristic Approaches for Fresh Produce Inventory Control and Shelf Space Allocation", Journal of the Operational Research Society, 59(10), pp. 1387-1397, 2007.

[8] J. L. Casti, "Firm Forecast", New Scientist, pp. 42-46, April 24, 1999.

[9] Y. Kubera, P. Mathieu, S. Picault, "Interaction-Oriented Agent Simulations: From Theory to Implementation", 18th European Conference on Artificial Intelligence (ECAI'08), pp. 383-387, IOS Press, 2008.

[10] Y. Kubera, P. Mathieu, S. Picault, "Everything can be Agent!", 9th International Joint Conference on Autonomous Agents and Multi-Agent Systems (AAMAS'10), 2010. 